\title{
Correlation between SYNTAX Score and Pattern of Risk Factors in Patients Referred for Coronary Angiography in Cardiology Department, Menoufia University
}

\author{
Ahmed Mokhtar El Kersh' ${ }^{*}$, Ahmed Ashraf Reda1, Mohamed Gamal El Hadad2, \\ Khaled Hussein El-Sharnouby ${ }^{2}$
}

${ }^{1}$ Department of Cardiology, Menoufia University Hospitals, Menoufia, Egypt

${ }^{2}$ Department of Cardiology, Shibin El Kom Teaching Hospital, Menoufia, Egypt

Email: *ahmedelkersh0@gmail.com

How to cite this paper: El Kersh, A.M., Reda, A.A., El Hadad, M.G. and El-Sharnouby, K.H. (2018) Correlation between SYNTAX Score and Pattern of Risk Factors in Patients Referred for Coronary Angiography in Cardiology Department, Menoufia University. World Journal of Cardiovascular Diseases, 8, 431-439.

https://doi.org/10.4236/wjcd.2018.88042

Received: June 14, 2018

Accepted: August 28, 2018

Published: August 31, 2018

Copyright $\odot 2018$ by authors and Scientific Research Publishing Inc. This work is licensed under the Creative Commons Attribution International License (CC BY 4.0).

http://creativecommons.org/licenses/by/4.0/

(c) (i) Open Access

\begin{abstract}
Objectives: The role of SYNTAX (SX) score in assessing the complexity of coronary artery disease (CAD) is well established. In this study we investigate the relationship between conventional risk factors of CAD with its complexity using SX score. Methods: The study consisted of 52 patients with CAD who were admitted to Cardiology Department-Menoufia University Hospitals for elective coronary angiography. The overall SX score was calculated prospectively using the SX score algorithm. Then comparison was done between populations with and without each risk factor. Results: There was a statistically significant correlation between age, hypertension, diabetes mellitus, dyslipidemia and smoking with SX score results $(\mathrm{p}<0.05)$ and an insignificant correlation regarding body mass index and gender $(\mathrm{p}>0.05)$. In a multivariate regression analysis, including conventional risk factors of $\mathrm{CAD}$ as independent covariates, it revealed that aging, having diabetes mellitus and smoking were identified as significant independent risk factors for CAD complexity. Conclusion: Advanced age, having diabetes mellitus and cigarette smoking are considered to be independent risk factors for the complexity of CAD. Therefore, when these factors present, we expect that the SX score of the patient to be high indicating a complex CAD.
\end{abstract}

\section{Keywords}

Coronary Artery Disease, SYNTAX Score, Risk Factors 


\section{Introduction}

The SYNTAX score (SX score) is a comprehensive angiographic scoring system that is derived entirely from the coronary anatomy and lesion characteristics [1] [2] [3]. It was initially designed to quantify lesion complexity; however, it is also able to predict major adverse cardiac events (MACE) after percutaneous revascularization in patients with multivessel coronary artery disease [4] [5] [6] and/or left main disease [7].

Previous studies pointed to the predictive ability of SX score for periprocedural myocardial infarction (MI) in patients undergoing elective percutaneous coronary intervention [8]. In the past few years it was proven that treatment and modification of different conventional risk factors helps in prevention of CAD. The most important finding was that aggressive medical therapy will lead to reduction of the likelihood of recurrent MACE in patients with established CAD (secondary prevention). The American Heart Association (AHA) and the American College of Cardiology (ACC) have published joint recommendations for medical intervention in patients with CAD and other forms of atherosclerotic disease [9].

However a large variability exists in risk status of patients without established CAD. This mandates a range of the intensity of interventions. Thus a comprehensive risk assessment is essential for patient categorization and proper selection of therapeutic intervention. The present statement was being published jointly by the AHA and ACC to outline current issues and approaches to global risk assessment for primary prevention. The approaches described in that statement can be used for guidance at several levels of primary prevention; however, the statement did not attempt to specifically link risk assessment to treatment guidelines for particular risk factors. Nonetheless, it provided critical background information that can be used in the development of new treatment guidelines [10].

The major and independent risk factors for CAD are cigarette smoking of any amount, systemic hypertension, dyslipidemia, diabetes mellitus, and advancing age. The relationship between these risk factors and CAD has been investigated by the Framingham Heart Study and other studies. These studies showed that the major risk factors are additive in predictive power. Accordingly, the total risk of a person can be estimated by a summing of the risk imparted by each of the major risk factors [11].

\section{Methods}

\subsection{Study Design}

This is a retrospective cohort study that included consecutive adult patients > 18 years of age admitted to Cardiology Department-Menoufia University Hospitals in order to investigate risk factors for the complexity of coronary artery disease using SYNTAX scores. 


\subsection{Study Population}

All patients $>18$ years of age admitted with established CAD and not having any of the exclusion criteria were included in the study. Patients with history of cigarette smoking of any amount, elevated blood pressure, diabetes mellitus, and advancing age were included in the study. Patients with elevated serum total cholesterol, elevated low-density lipoprotein cholesterol (LDL-C), and low serum high-density lipoprotein cholesterol (HDL-C) in lipogram were also included in the study. While patients with acute MI or past history of previous percutaneous coronary intervention and coronary artery bypass graft were excluded from the study.

All patients were subjected to informed consent, thorough history taking and clinical examination, with special concern on history of smoking, history of chronic diseases like diabetes and hypertension history of previous coronary angiography or previous PCI, and history of previous myocardial infarction. They were also subjected to elective coronary angiography. From the baseline diagnostic angiogram, each coronary lesion producing $\geq 50 \%$ diameter stenosis in vessels $\geq 1.5 \mathrm{~mm}$ will be scored separately and added together to provide the overall SX score, which was calculated prospectively using the SX score algorithm.

\subsection{Statistical Analysis}

Data were collected, revised and edited into a master table using Microsoft Excel 2013. Data were then revised, coded and entered to the statistical package for social science (SPSS) version 22. Patient demographic characteristics were presented as mean and standard deviation for continuous variables with normal distribution, median and interquartile range (IQR) for continuous variables with non-normal distributions and as proportions (percentages) for categorical variables. For normally distributed data, comparison between two independent populations was done using independent t-test. For abnormally distributed data, comparison between two independent populations was done using Mann Whitney $\mathrm{U}$ test. The distributions of quantitative variables were tested for normality using Kolmogorov-Smirnov test, Shapiro-Wilk test and D'Agstino test, also Histogram and QQ plot were used for visual assessment. If it reveals normal data distribution, parametric tests (e.g. Pearson's correlation) were applied. If the data were abnormally distributed, non-parametric (e.g. Kendall's tau-b correlation) tests were used. Univariate and multivariate regression analyses were conducted between SX score as a dependent variable and each of the following as independent variables; age, gender, HTN, DM, smoking, dyslipidemia and BMI. The confidence interval was set to $95 \%$ and the margin of error accepted was set to $5 \%$. So a p value lower than 0.05 was considered significant.

\section{Results}

The study population consisted of 52 consecutive patients with established CAD. 
In all, $31(59.6 \%)$ of the patients were male, and mean age of patients with its SD was $54.3 \pm 9.61$ years. In all, 31 (59.6\%) patients were hypertensive, 18 (34.6\%) patients were diabetic, $22(42.3 \%)$ patients were dyslipidemic, and 20 (38.5\%) patients were smokers. The patients BMI had a median value and IQR of 25 (24, 26) $\mathrm{kg} / \mathrm{m}^{2}$ with a maximal BMI of $39 \mathrm{~kg} / \mathrm{m}^{2}$ and a minimal BMI of $23 \mathrm{~kg} / \mathrm{m}^{2}$. Regarding SX score, the median value was 15 with an (IQR) of $(10,22)$, a minimum value of 10 and a maximum value of 33 (Table 1 ).

A Pearson's correlation was run to assess the relationship between patient's age and their SX score. There was a strong positive correlation between age and SX score, $(r(50)=0.639, \mathrm{p}<0.001)$, with age explaining $40 \%$ of the variation in SX score (Figure 1). There was insignificant difference between male and female populations regarding SX score. The SX score showed a median value of 18 with an IQR of $(12,25)$ in male patients vs. $15(10,21)$ in female patients $(p=0.065)$, (Table 2). The mean and SD of SX score was $20 \pm 7.2$ in hypertensive patients vs. a median result of 10 and IQR of $(10,15)$ in non-hypertensive patients ( $p=$ $0.003)$. While the mean and SD of SX score was $24.4 \pm 5.5$ in diabetic patients vs. $12(10,18)$ as median and IQR in non-diabetic patients $(\mathrm{p}<0.001)$. As regard dyslipidemia, the mean and SD of SX score was $22.4 \pm 6.1$ in dyslipidemic patients vs. $11(10,15.8)$ as median and IQR in non-dyslipidemic patients ( $\mathrm{p}<$ $0.001)$. In relation to smoking, the mean and SD of SX score was $20.1 \pm 7.3$ in smokers vs. $15(10,21.5)$ as median and IQR in non-smokers $(p=0.006)$ (Table 2). A Kendall's tau-b correlation was run to assess the relationship between patients BMI and their SX scores. There was a weak positive correlation between BMI and SX score, $\mathrm{r}(50)=0.182, \mathrm{p}=0.091$ (Figure 2).

A univariate and multivariate regression analyses were conducted using SX score as a dependent variable and each of the following factors as independent variables; age, gender, HTN, DM, smoking, dyslipidemia and BMI. It revealed that aging, having diabetes mellitus and smoking were identified as significant independent risk factors (age: multiple regression coefficient $0.3, \mathrm{p}<0.001$; diabetes mellitus: 6.7, $\mathrm{p}<0.001$; smoking: 5.8, $\mathrm{p}<0.001$ ). However, being a male, hypertension, dyslipidemia and BMI were not statistically significant risk factors for $\mathrm{CAD}$ complexity. Among these independent factors, diabetes mellitus was the strongest risk factor (multiple regression coefficient: 6.7, $\mathrm{p}<0.001$ ) (Table 3).

Table 1. Baseline characteristics.

\begin{tabular}{cc}
\hline Variable & Distribution \\
\hline Age [years] & $54.3 \pm 9.61$ \\
Male gender [No. (\%)] & $31(59.6 \%)$ \\
DM [No. (\%)] & $18(34.6 \%)$ \\
HTN [No. (\%)] & $31(59.6 \%)$ \\
Dyslipidemia [No. (\%)] & $22(42.3 \%)$ \\
Smoking [No. (\%)] & $20(38.5 \%)$ \\
BMI [kg/m $^{2}$ & $25(24,26)$ \\
SYNTAX score & $15(10,22)$ \\
\hline
\end{tabular}

Abbreviations: HTN, hypertension; DM, diabetes mellitus; BMI, body mass index. 
Table 2. SX score comparison between populations with and without each risk factor.

\begin{tabular}{cccc}
\hline Variable & $\begin{array}{c}\text { SX score in patients with } \\
\text { each risk factor }\end{array}$ & $\begin{array}{c}\text { SX score in patients } \\
\text { without each risk factor }\end{array}$ & $\mathrm{p}$ \\
\hline Male gender & $\mathrm{n}=31($ male $)$ & $\mathrm{n}=21$ (female) & 0.065 \\
& $18(12,25)$ & $15(10,21)$ & \\
HTN & $\mathrm{n}=31$ & $\mathrm{n}=21$ & 0.003 \\
& $20 \pm 7.2$ & $10(10,15)$ & \\
DM & $\mathrm{n}=18$ & $\mathrm{n}=34$ & \\
& $24.4 \pm 5.5$ & $12(10,18)$ & $<0.001$ \\
HPL & $\mathrm{n}=22$ & $\mathrm{n}=30$ & \\
& $22.4 \pm 6.1$ & $11(10,15.8)$ & 0.006 \\
Smoker & $\mathrm{n}=20$ & $\mathrm{n}=32$ & \\
\hline
\end{tabular}

Abbreviations: HTN, hypertension; DM, diabetes mellitus; HPL, hyperlipidemia. Data are expressed as mean $\pm S D$, median and IQR, or number (percentage).

Table 3. Univariate and multivariate regression analyses of risk factors for SYNTAX score.

\begin{tabular}{ccccc}
\hline \multirow{2}{*}{ Variable } & \multicolumn{2}{c}{ Univariate regression } & \multicolumn{2}{c}{ Multivariate regression } \\
\cline { 2 - 5 } & $\begin{array}{c}\text { Coefficient } \& \\
95 \% \text { CI }\end{array}$ & $\mathrm{p}$ & $\begin{array}{c}\text { Coefficient } \& \\
95 \% \mathrm{CI}\end{array}$ & $\mathrm{p}$ \\
\hline Age & $0.47(0.31$ to 0.63$)$ & $<0.001$ & $0.32(0.19$ to 0.45$)$ & $<0.001$ \\
Male gender & $-3.6(-7.5$ to 0.33$)$ & 0.072 & $1.35(-1.7$ to 4.4$)$ & 0.383 \\
HTN & $6(2.3$ to 9.7$)$ & $\mathbf{0 . 0 0 2}$ & $0.25(-2.2$ to 2.7$)$ & 0.83 \\
DM & $10.5(7.6$ to 13.4$)$ & $<0.001$ & $6.7(3.3$ to 10.1$)$ & $<0.001$ \\
HPL & $8.3(5$ to 11.6$)$ & $<0.001$ & $0.3(-3$ to 3.6$)$ & 0.851 \\
Smoking & $5.4(1.6$ to 9.2$)$ & $\mathbf{0 . 0 0 6}$ & $5.8(2.9$ to 8.7$)$ & $<0.001$ \\
BMI & $0.5(-0.2$ to 1.2$)$ & 0.157 & $0.13(-0.32$ to 0.58$)$ & 0.569 \\
\hline
\end{tabular}

Abbreviations: HTN, hypertension; DM, diabetes mellitus; HPL, hyperlipidemia; BMI, body mass index.

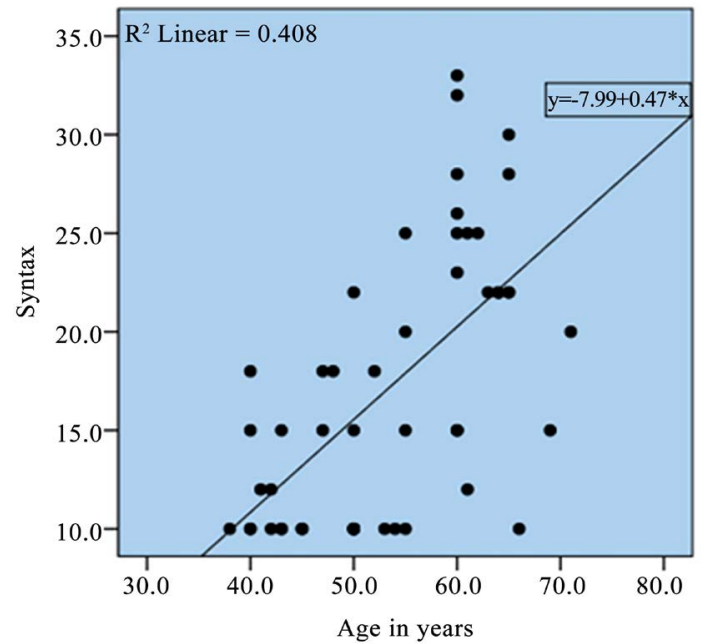

Figure 1. A scatterplot of age against SYNTAX score among the study population. 


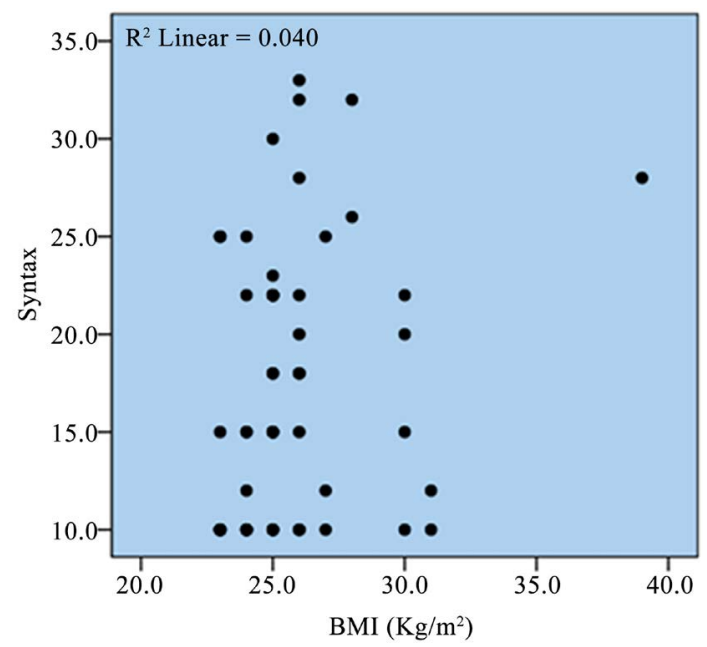

Figure 2. A scatterplot of BMI against SYNTAX score among the study population.

\section{Discussion}

The SYNTAX trial is the origin from which the SX score was developed. It was used as an index for assessment of lesion complexity in patients with left main trunk lesion or three-vessel disease for whom CABG treatment is indicated [1] [5] [12] [13]. Conventional risk factors of CAD such as age, male gender, hypertension, dyslipidaemia, BMI, diabetes and smoking have been linked to more severe forms of CAD [11]. A higher patient's risk status is expected to be accompanied by a more severe and advanced form of the disease and a higher SX score. The evidence regarding such statement is still not that sufficient [14].

The aim of this work was to study the correlation between SX score and pattern of risk factors in patients referred for coronary angiography in Cardiology Department-Menoufia University. The study enrolled 52 cardiac patients. All patients were subjected to history taking, full clinical examination, angiographic analysis and SX score. The data was collected and statistically analyzed.

In this study, aging, having diabetes mellitus and smoking were significant independent risk factors of the complexity of CAD and high SX score results. So, patients with these factors are expected to have a more complicated CAD than that in patients without these factors. In the SYNTAX trial, rates of MACE and cerebrovascular events were similar in patients with low or intermediate scores in both the CABG group as well as in the PCI group. However this wasn't the case in patients with high scores, in which, patients in the PCI group had significantly increased event rate [5] [12] [13]. Based on this finding, it is recommended to avoid PCI in patients with high SX score as a result of expected high complexity of CAD in those patients, and this is also why CABG should be considered.

It is known that vascular endothelium function decreases and arterial sclerosis progresses with aging [15]. In our study, it was found that advanced age is an independent risk factor of the complexity of CAD.

In this study, being a male wasn't a statistically significant risk factor for the 
complexity of CAD. It is known from a previous study that estrogen shows antiarteriosclerotic effects and that's why women develop CAD after menopause [16]. It was previously thought that CAD in men is more complex and severe than in women. However, several studies reported that women with CAD have a smaller reference diameter of coronary artery, as well as more frequent diffuse stenosis not suitable for PCI, calcification and coronary artery dissection than men [17] [18] [19] [20]. Besides, it was also found in a previous study that except for female hormonal status, no risk factor has been recognized as acting on one gender and not on the other. This finding indicates that the pathogenesis of CAD is very similar for men and women [21].

It is now universally well established that cigarette smoke exposure is an important cause of cardiovascular morbidity and mortality. This is because either active or passive exposure leads to vasomotor dysfunction, atherogenesis, and thrombosis in multiple vascular beds [22]. In our study smoking is found to be an independent risk factor of the complexity of CAD.

Diabetes mellitus is found to be associated with the complexity of CAD [23]. In our study, we found that diabetes mellitus is an independent risk factor for the complexity of CAD.

Lastly, in this study, the conventional risk factors of CAD were not totally concordant with those of CAD complexity. We found that factors, such as male gender, hypertension, increased BMI and hypercholesterolemia were not significant independent risk factors for the complexity of CAD.

\section{Study Limitations}

Although the number of patients enrolled in our study is relatively small, the results are quite comparable to larger studies. This study was observational and single-institutional in nature, which possibly restricted us from identifying and analyzing all potential confounding factors. There was some sort of selection bias because the choice between PCI and CABG as a treatment is dependent on the treating physician. Exclusion of acute MI because SX score is not yet valid in such condition, is considered one of the study limitations. Lastly, we didn't demonstrate the effect of treating different modifiable risk factors on CAD lesion complexity.

\section{Conclusion}

Regarding conventional risk factors of CAD, being an advanced age, having diabetes mellitus and cigarette smoking are considered to be independent risk factors for the complexity of CAD. Therefore, presence of these risk factors will lead to the expectation of a highly complex CAD which in turn requires a careful management and a more tendency for CABG as a method of revascularization. Further studies in larger cohorts are needed for the validation of these findings to better define the role of conventional risk factors in clinical decision making in patients with CAD. 


\section{Declaration of Conflicting Interests}

The author(s) declared no potential conflicts of interest with respect to the research, authorship, and/or publication of this article.

\section{Funding}

The author(s) received no financial support for the research, authorship, and/or publication of this article.

\section{References}

[1] Sianos, G., Morel, M.A., Kappetein, A.P., et al. (2005) The SYNTAX Score: An Angiographic Tool Grading the Complexity of Coronary Artery Disease. Eurointervention, 1, 219-227.

[2] Serruys, P.W., Onuma, Y., Garg, S., et al. (2009) Assessment of the SYNTAX Score in the Syntax Study. Eurointervention, 5, 50-56. https://doi.org/10.4244/EIJV5I1A9

[3] SYNTAX Working Group SYNTAX Score Calculator. http://www.syntaxscore.com

[4] Valgimigli, M., Serruys, P.W., Tsuchida, K., et al. (2007) Cyphering the Complexity of Coronary Artery Disease Using the Syntax Score to Predict Clinical Outcome in Patients with Three-Vessel Lumen Obstruction Undergoing Percutaneous Coronary Intervention. The American Journal of Cardiology, 99, 1072-1081.

https://doi.org/10.1016/j.amjcard.2006.11.062

[5] Serruys, P.W., Morice, M.C., Kappetein, A.P., et al. (2009) Percutaneous Coronary Intervention versus Coronary-Artery Bypass Grafting for Severe Coronary Artery Disease. The New England Journal of Medicine, 360, 961-972. https://doi.org/10.1056/NEJMoa0804626

[6] Serruys, P.W., Onuma, Y., Garg, S., et al. (2010) 5-Year Clinical Outcomes of the ARTS II (Arterial Revascularization Therapies Study II) of the Sirolimus-Eluting Stent in the Treatment of Patients with Multivessel De Novo Coronary Artery Lesions. Journal of the American College of Cardiology, 55, 1093-1101. https://doi.org/10.1016/j.jacc.2009.11.049

[7] Capodanno, D., Di Salvo, M.E., Cincotta, G., Miano, M., Tamburino, C. and Tamburino, C. (2009) Usefulness of the SYNTAX Score for Predicting Clinical Outcome after Percutaneous Coronary Intervention of Unprotected Left Main Coronary Artery Disease. Circulation: Cardiovascular Interventions, 2, 302-308. https://doi.org/10.1161/CIRCINTERVENTIONS.108.847137

[8] van Gaal, W.J., Ponnuthurai, F.A., Selvanayagam, J., et al. (2009) The Syntax Score Predicts Peri-Procedural Myocardial Necrosis during Percutaneous Coronary Intervention. International Journal of Cardiology, 135, 60-65. https://doi.org/10.1016/j.ijcard.2008.03.033

[9] Smith, S.C., Blair, S.N., Criqui, M.H., et al. (1995) Preventing Heart Attack and Death in Patients with Coronary Disease. Journal of the American College of Cardiology, 26, 292. https://doi.org/10.1016/0735-1097(95)90846-G

[10] Grundy, S.M., Pasternak, R., Greenland, P., Smith, S. and Fuster, V. (1999) Assessment of Cardiovascular Risk by Use of Multiple-Risk-Factor Assessment Equations: A Statement for Healthcare Professionals from the American Heart Association and the American College of Cardiology. Circulation, 100, 1481-1492. https://doi.org/10.1161/01.CIR.100.13.1481

[11] Wilson, P.W., D’Agostino, R.B., Levy, D., Belanger, A.M., Silbershatz, H. and Kan- 
nel, W.B. (1998) Prediction of Coronary Heart Disease Using Risk Factor Categories. Circulation, 97, 1837-1847. https://doi.org/10.1161/01.CIR.97.18.1837

[12] Mohr, F.W., Rastan, A.J., Serruys, P.W., et al. (2011) Complex Coronary Anatomy in Coronary Artery Bypass Graft Surgery: Impact of Complex Coronary Anatomy in Modern Bypass Surgery? Lessons Learned from the SYNTAX Trial after Two Years. The Journal of Thoracic and Cardiovascular Surgery, 141, 130-140. https://doi.org/10.1016/j.jtcvs.2010.07.094

[13] Kappetein, A.P., Feldman, T.E., Mack, M.J., et al. (2011) Comparison of Coronary Bypass Surgery with Drugeluting Stenting for the Treatment of Left Main and/or Three-Vessel Disease: 3-Year Follow-Up of the SYNTAX Trial. European Heart Journal, 32, 2125-2134. https://doi.org/10.1093/eurheartj/ehr213

[14] Tanaka, T., Seto, S., Yamamoto, K., Kondo, M. and Otomo, T. (2013) An Assessment of Risk Factors for the Complexity of Coronary Artery Disease Using the SYNTAX Score. Cardiovascular Intervention and Therapeutics, 28, 16-21. https://doi.org/10.1007/s12928-012-0112-5

[15] Celermajer, D.S., Sorensen, K.E., Spiegelhalter, D.J., Georgakopoulos, D., Robinson, J. and Deanfield, J.E. (1994) Aging Is Associated with Endothelial Dysfunction in Healthy Men Years before the Age-Related Decline in Women. Journal of the American College of Cardiology, 24, 471-476. https://doi.org/10.1016/0735-1097(94)90305-0

[16] Mendelsohn, M.E. and Karas, R.H. (1999) The Protective Effects of Estrogen on the Cardiovascular System. The New England Journal of Medicine, 340, 1801-1811. https://doi.org/10.1056/NEJM199906103402306

[17] Nakamura, T., Ogita, M., Ako, J. and Momomura, S. (2010) Gender Differences of Plaque Characteristics in Elderly Patients with Stable Angina Pectoris: An Intravascular Ultrasonic Radiofrequency Data Analysis. International Journal of Vascular Medicine, 2010, Article ID: 134692. https://doi.org/10.1155/2010/134692

[18] Jacobs, A.K. (2003) Coronary Revascularization in Women in 2003; Sex Revisited. Circulation, 107, 375-377. https://doi.org/10.1161/01.CIR.0000048895.18974.6B

[19] Kornowski, R., Lansky, A.J., Mintz, G.S., et al. (1997) Comparison of Men versus Women in Cross-Sectional Area Luminal Narrowing, Quantity of Plaque, Presence of Calcium in Plaque, and Lumen Location in Coronary Arteries by Intravascular Ultrasound in Patients with Stable Angina Pectoris. American Journal of Cardiology, 79, 1601-1605. https://doi.org/10.1016/S0002-9149(97)00206-3

[20] Inoue, F., Yamaguchi, S., Ueshima, K., et al. (2010) Gender Differences in Coronary Plaque Characteristics in Patients with Stable Angina: A Virtual Histology Intravascular Ultrasound Study. Cardiovascular Intervention and Therapeutics, 25, 40-45. https://doi.org/10.1007/s12928-009-0009-0

[21] Roeters van Lennep, J.E., Westerveld, H.T., Erkelens, D.W. and van der Wall, E.E. (2002) Risk Factors for Coronary Heart Disease: Implications of Gender. Cardiovascular Research, 53, 538-549. https://doi.org/10.1016/S0008-6363(01)00388-1

[22] Ambrose, J.A. and Barua, R.S. (2004) The Pathophysiology of Cigarette Smoking and Cardiovascular Disease: An Update. Journal of the American College of Cardiology, 43, 1731-1737. https://doi.org/10.1016/j.jacc.2003.12.047

[23] Baris, N., Akdeniz, B., Uyar, S., et al. (2006) Are Complex Coronary Lesions More Frequent in Patients with Diabetes Mellitus? Canadian Journal of Cardiology, 22, 935-937. https://doi.org/10.1016/S0828-282X(06)70312-9 